\title{
High-Frequency Oscillation and Recovery Functions of Somatosensory Evoked Potentials in Human T-Cell Lymphotropic Virus Type 1-Associated Myelopathy
}

Nobuyuki Ishii, Hitoshi Mochizuki ${ }^{*}$, Kazutaka Shiomi and Masamitsu Nakazato

Department of Internal Medicine, University of Miyazaki, Miyazaki, Japan

*Corresponding author: Hitoshi Mochizuki, Division of Neurology, Respirology, Endocrinology and Metabolism, Department of Internal Medicine, University of Miyazaki, 5200 Kihara, Kiyotake, Miyazaki, 889-1692, Japan, Tel: +81-985-85-2965, Fax: +81-985-85-1869; E-mail: mochizuki-h@umin.ac.jp

Received date: Nov 05, 2014, Accepted date: Dec 06, 2014, Published date: Dec 12, 2014

Copyright: @ 2014 Ishii N, et al. This is an open-access article distributed under the terms of the Creative Commons Attribution License, which permits unrestricted use, distribution, and reproduction in any medium, provided the original author and source are credited.

\begin{abstract}
Objectives: Human T-cell lymphotropic virus type 1-associated myelopathy (HAM) involves not only spinal cord but also the cerebral cortex and the white matter in histopathological analysis. In addition, a previous study found that T2 hyperintensities on brain magnetic resonance imaging (MRI) in HAM patients were increased only in white matter. However, conventional neurophysiological examination of somatosensory pathway did not identify any abnormalities in the cerebral cortex or white matter. We evaluated the inhibitory function of the cerebral sensory cortex in HAM patients by analyzing high-frequency oscillations (HFOs) and somatosensory evoked potential recovery functions (SEP-Rs).
\end{abstract}

Method: Eight HAM patients were enrolled in the present investigation. Twenty age-matched healthy subjects were enrolled (10 for HFOs, 10 for SEP-Rs). SEP was recorded from the hand sensory area contralateral to the median nerve stimulated at the wrist. HFOs were obtained by digitally filtering raw SEPs from 500 to $1000 \mathrm{~Hz}$. We measured amplitudes of the N20 onset-peak (N20o-p), N20 peak-P25 peak (N20p-P25p), P25 peak-N33 peak (P25p-N33p), and the early (1st-2nd) and late (3rd-4th) HFOs. For recovery function study, paired-pulse stimuli at various interstimulus intervals (ISI; 20 - $200 \mathrm{~ms}$ ) were given.

Results: None of the SEP components, and neither early nor late HFOs, showed any significant differences between HAM patients and normal controls. In recovery function study, there were no significant differences in any of the components between the two groups, although the HAM group had a tendency to disinhibit in the recovery curve for N20o-p amplitudes $(P=0.075)$.

Conclusions: Normal early and late HFOs indicate that both basal ganglia and GABAergic inhibitory interneuron activity in the cortex are intact in HAM patients. The recovery function findings might indicate mild impairment of subcortical white matter in HAM patients because disinhibition of N20o-p and normal inhibition of the N20p-P25p pattern were previously observed in Binswanger's disease. Our conclusions are: (1) HAM patients may demonstrate mild subcortical dysfunction as previously found on MRI. (2) Sensory cortical function is normal in HAM patients.

Keywords: HTLV-1-associated myelopathy; Somatosensory evoked potential; High-frequency oscillation; Recovery function; Sensory cortex; White matter; Interneuron; Human T-cell lymphotropic virus type 1

\section{Introduction}

Human T-cell lymphotropic virus type 1-associated myelopathy (HAM) primary involves the spinal cord, especially the thoracic cord [1]. In histopathological analysis, inflammation is observed in the cerebral cortex and the white matter as well as in the spinal cord [2]. A previous study found that T2 hyperintensities on brain magnetic resonance imaging (MRI) in HAM patients were increased only in white matter [3], although few reports have studied the cerebral cortex. On the other hand, conventional neurophysiological examination of somatosensory function did not identify any abnormalities in the cerebral cortex or white matter [4]. In this paper we investigated for the first time the inhibitory function of the cerebral sensory cortex in HAM patients by analyzing both high-frequency oscillations (HFOs) derived from cerebral cortex and somatosensory evoked potential recovery functions (SEP-Rs). HFOs are several brief inflections in the range of $600-900 \mathrm{~Hz}$ superimposed mainly on the ascending slope of the N20 primary cortical response following stimulation of the median nerve [5]. Early HFOs have some relation with the basal ganglia function, whereas late HFOs reflect GABAergic inhibitory interneuron activity in the cortex [5-7]. By measuring SEP amplitudes by paired-pulse stimuli on a peripheral nerve, SEP-Rs can show changes in excitability of the somatosensory cortex in patients who showed no conduction delays in conventional SEPs [8]. Combination of both methods could detect slight abnormalities in the cerebral cortex and the white matter.

\section{Subjects and Methods}

\section{Subjects}

The subjects were eight patients (seven women and one man, aged 42-80 years) with HAM. The diagnosis of HAM was made according to the World Health Oraganization diagnostic criteria [9]. The median Expanded Disability Status Score [10] (EDSS) and Osame's Motor Disability Score [11] (OMDS) were 6 (range 4-7) and 5 (range 4-10), 
Citation: Ishii N, Mochizuki H, Shiomi K, Nakazato M (2014) High-Frequency Oscillation and Recovery Functions of Somatosensory Evoked Potentials in Human T-Cell Lymphotropic Virus Type 1-Associated Myelopathy. J Neurol Neurophysiol 5: 254. doi: $10.4172 / 2155-9562.1000254$

Page 2 of 4

respectively. The median duration of illness was seven years (range 113 years). Six of eight patients had normal brain MRIs, while the other two had a few small T2 hyperintense lesions in the deep white matter. As normal controls, 20 age-matched healthy subjects were enrolled (10 for HFOs, 10 for SEP-Rs). All subjects agreed to participate in the study and signed an informed consent form approved by the institutional review board of the University of Miyazaki.

\section{Somatosensory evoked potentials}

Somatosensory evoked potentials (SEPs) were recorded from the ipsilateral Erb's point and contralateral hand sensory area after right median nerve stimulation at the wrist. A recording electrode was placed over C3' (2 cm posterior to the C3 placement on the International 10-20 System) and right Erb's point, with a midfrontal (Fz) reference. Potentials were amplified with filters set at 1 and 2000 $\mathrm{Hz}$, and at least 256 responses were averaged. Stimuli were applied with a repetition rate of $3 \mathrm{~Hz}$ and the intensity was fixed at about 1.2 times the motor threshold. We monitored the N9 amplitude to confirm that the appropriate stimulus intensity was reached. HFOs were obtained by digitally filtering raw SEPs (using fast reversed Fourier transformation) from 500 to $1000 \mathrm{~Hz}$ (Figure 1a) [6]. We measured amplitudes of the N20 onset-peak (N20o-p), N20 peak-P25 peak (N20p-P25p), P25 peak-N33 peak (P25p-N33p), and the early (1st-2nd) and late (3rd-4th) HFOs.

SEP-Rs were studied using a paired stimulation technique. The paired stimuli (S1 and S2) of equal intensity were administered at various interstimulus intervals (ISI; 20, 40,60,80, 100, 150, and 200 $\mathrm{ms}$ ). Each single stimulus or stimulus pair was applied with a repetition rate of $0.8 \mathrm{~Hz}$. Other SEP settings were the same as those used in conventional SEPs and HFOs. SEPs evoked by the second stimulus (SEP2) were obtained by subtracting SEPs evoked by S1 alone (SEP1) from those elicited by paired stimuli (SEP1+SEP2) [8]. SEP1, SEP1+2, and SEP2 are shown in Figure $1 b$. a

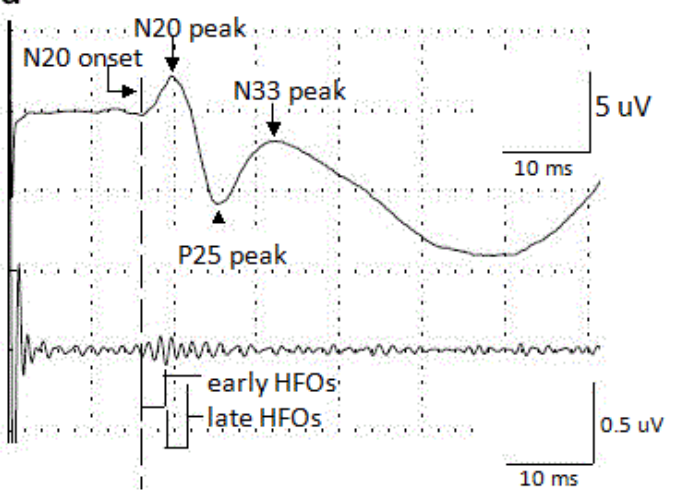

b

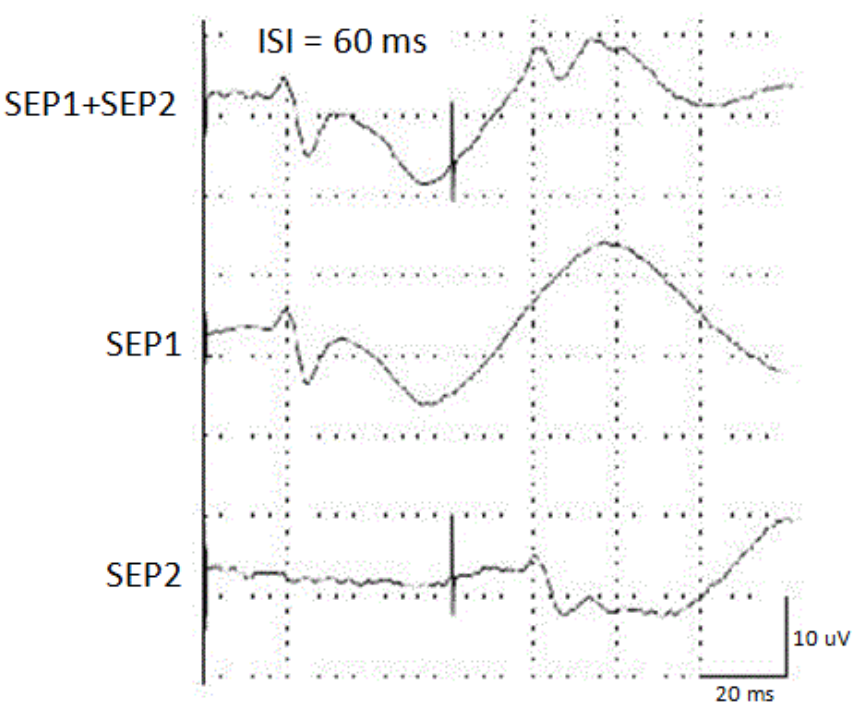

Figure 1: (a) The top row shows SEPs elicited by single-pulse stimulation, and the bottom SEPs were digitally filtered at $500-1000 \mathrm{~Hz}$. (b) The top row shows SEPs (SEP1+SEP2) elicited by paired-pulse stimulation (ISI $=60 \mathrm{~ms}$ ), and SEP1 are elicited by single-pulse stimulation. SEP2 are obtained by subtracting SEP1 from SEP1+SEP2.

\begin{tabular}{|l|l|l|l|l|l|}
\hline & \multicolumn{2}{|l|}{ Normal controls } & \multicolumn{2}{l|}{ HAM patients } & \\
\hline Amplitude $(\mu \mathrm{V})$ & Mean & SD & Mean & SD & P-value \\
\hline N20o-p & 2.4 & 1.1 & 2.7 & 1.1 & 0.82 \\
\hline N20p-P25p & 5.0 & 2.6 & 7.0 & 3.2 & 0.50 \\
\hline P25p-N33p & 3.6 & 2.5 & 4.0 & 2.5 & 0.55 \\
\hline Early HFOs & 0.27 & 0.23 & 0.22 & 0.14 & 0.56 \\
\hline Late HFOs & 0.26 & 0.15 & 0.28 & 0.09 & 0.82 \\
\hline
\end{tabular}

We compared the amplitudes of the three conventional SEP components (N20o-p, N20p-P25p, P25p-N33p) and the two HFO components (early and late) in both groups using an unpaired t-test. To overcome inter-subject variability in the absolute amplitudes of these components when analyzing recovery functions, we used the ratios of the three parameters (N20o-p, N20p-P25p, P25p-N33p) evoked by a paired-pulse stimulation at every ISI to those evoked by a single-pulse stimulation. We evaluated the time courses of recovery functions by plotting the grand means $( \pm S D)$ of these ratios against ISIs. For each parameter, we compared time courses using repeated ANOVA with the Scheffe method for post hoc analysis.

Table 1: Amplitudes of raw SEPs and HFOs; Comparisons were performed with unpaired t-test analysis.

\section{Statistical analysis}

\section{Results}

Amplitudes of each SEP component and the HFOs of both groups are shown in Table 1 . None of the SEP components, and neither early 
Citation: Ishii N, Mochizuki H, Shiomi K, Nakazato M (2014) High-Frequency Oscillation and Recovery Functions of Somatosensory Evoked Potentials in Human T-Cell Lymphotropic Virus Type 1-Associated Myelopathy. J Neurol Neurophysiol 5: 254. doi: $10.4172 / 2155-9562.1000254$

Page 3 of 4

nor late HFOs, showed any significant differences between HAM patients and normal controls.

Figure 2 shows the mean $( \pm \mathrm{SD})$ recovery curves of the three components for HAM patients (solid line) and normal controls (dashed line). Repeated ANOVA revealed no significant differences in any of the components between the two groups, although the HAM group had a tendency to disinhibit in the recovery curve for the N20o$\mathrm{p}$ amplitudes $(\mathrm{P}=0.075)$.
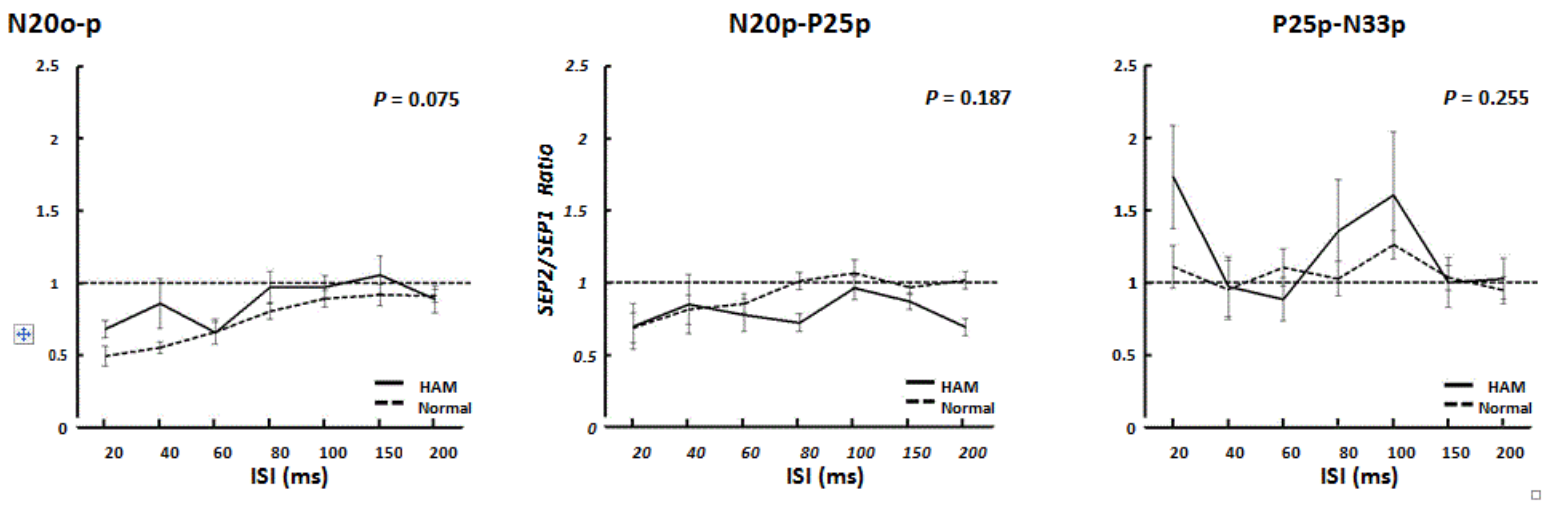

Figure 2: Mean $( \pm S D)$ recovery curves of three components (N20o-p, N20p-P25p, and P25p-N33p) in HAM patients (solid line) and normal controls (dashed line) are shown. There were no significant differences in the recovery functions between the two groups, although the HAM group had a tendency to disinhibit in the recovery curve for $\mathrm{N} 20 \mathrm{o}-\mathrm{p}$ amplitudes $(\mathrm{P}=0.075)$. Comparisons were performed by repeated ANOVA with the Scheffe method for post hoc analysis.

\section{Discussion}

The results of conventional SEPs and HFOs in HAM patients did not differ significantly with those in normal controls. Conventional SEPs did not reveal any abnormalities in HAM patients, which confirm the findings of a previous study [4]. Neither early nor late HFOs differed significantly between HAM patients and normal controls. This is the first report on HFOs in patients with HAM. Early HFOs are suggested to be associated with the basal ganglia, whereas late HFOs reflect GABAergic inhibitory interneuron activity in the cortex $[6,7]$. Our study indicates that these functions are intact in HAM patients.

SEP-Rs did not differ significantly between the two groups. The N20o-p recovery curve in the HAM group, however, tended to be disinhibited. Abnormal inhibition of N20o-p and normal inhibition of the N20p-P25p pattern were previously observed in Binswanger's disease [12]. The recovery function findings in this study might indicate mild impairment of subcortical white matter in HAM patients.

This study suggested that HAM patients had slight abnormalities in subcortical function which could not be identified by a conventional neurophysiological examination. Small white matter lesions on brain MRI are frequently seen in subcortical and periventricular areas in patients with HAM [3], which is compatible with our results. The fact that only a small number of HAM patients were enrolled in this study, along with their mild disease severity, may have resulted in the lack of a significant difference in the N20o-p recovery function. We could not detect any cortical dysfunction in our study.

\section{Conclusion}

In neurophysiological analysis, HAM patients demonstrated mild subcortical dysfunction and normal sensory cortical function. This results are compatible with brain MRI abnormalities in HAM patients.

\section{References}

1. Iwasaki Y (1990) Pathology of chronic myelopathy associated with HTLV-I infection (HAM/TSP). J Neurol Sci 96: 103-123.

2. Aye MM, Matsuoka E, Moritoyo T, Umehara F, Suehara M, et al. (2000) Histopathological analysis of four autopsy cases of HTLV-I-associated myelopathy/tropical spastic paraparesis: inflammatory changes occur simultaneously in the entire central nervous system. Acta Neuropathol 100: 245-252.

3. Morgan DJ, Caskey MF, Abbehusen C, Oliveira-Filho J, Araujo C, et al. (2007) Brain magnetic resonance imaging white matter lesions are frequent in HTLV-I carriers and do not discriminate from HAM/TSP. AIDS Res Hum Retroviruses 23: 1499-1504.

4. Kakigi R, Shibasaki H, Kuroda Y, Endo C, Oda K, et al. (1988) Multimodality evoked potentials in HTLV-I associated myelopathy. J Neurol Neurosurg Psychiatry 51: 1094-1096.

5. Mochizuki H, Ugawa Y, Machii K, Terao Y, Hanajima R, et al. (1999) Somatosensory evoked high-frequency oscillation in Parkinson's disease and myoclonus epilepsy. Clin Neurophysiol 110: 185-191.

6. Mochizuki H, Machii K, Terao Y, Furubayashi T, Hanajima R, et al. (2003) Recovery function of and effects of hyperventilation on somatosensory evoked high-frequency oscillation in Parkinson's disease and myoclonus epilepsy. Neurosci Res 46: 485-492.

7. Ozaki I, Hashimoto I (2011) Exploring the physiology and function of high-frequency oscillations (HFOs) from the somatosensory cortex. Clin Neurophysiol 122: 1908-1923. 
Citation: Ishii N, Mochizuki H, Shiomi K, Nakazato M (2014) High-Frequency Oscillation and Recovery Functions of Somatosensory Evoked Potentials in Human T-Cell Lymphotropic Virus Type 1-Associated Myelopathy. J Neurol Neurophysiol 5: 254. doi: $10.4172 / 2155-9562.1000254$

Page 4 of 4

8. Mochizuki H, Masaki T, Matsushita S, Kamakura K, Motoyoshi K, et al. (2006) Disinhibition of somatosensory evoked potential recovery in alcoholics. Eur J Neurol 13: 896-900.

9. WHO (1989) Virus diseases: human T lymphotropic virus type I, HTLVI. Wkly Epidemiol Rec 64: 382-383.

10. Kurtzke JF (1983) Rating neurologic impairment in multiple sclerosis: an expanded disability status scale (EDSS). Neurology 33: 1444-1452.
11. Izumo S, Goto I, Itoyama Y, Okajima T, Watanabe S, et al. (1996) Interferon-alpha is effective in HTLV-I-associated myelopathy: a multicenter, randomized, double-blind, controlled trial. Neurology 46: 1016-1021.

12. Ugawa Y, Genba-Shimizu K, Kanazawa I (1996) Somatosensory evoked potential recovery (SEP-R) in various neurological disorders. Electroencephalogr Clin Neurophysiol 100: 62-67. 